\title{
Out of the Mouth of Babes: Lessons from Research on Human Infants
}

\author{
Stan A. Kuczaj II ${ }^{1}$ and Malin K. Lilley ${ }^{1^{*}}$ \\ ${ }^{1}$ Department of Psychology, The University of Southern Mississippi \\ *Corresponding author (Email: malin.lilley@usm.edu)
}

Citation - Kuczaj, S. A. II., \& Lilley, M. K. (2016). Out of the mouth of babes: Lessons from research on human infants. Animal Behavior and Cognition, 3(4), 212-223. doi: 10.12966/abc.02.11.2016

\begin{abstract}
Marine mammal behavior and cognition researchers often face a number of challenges, including the research subjects' lack of interest and verbal abilities, as well as choosing a paradigm with appropriate stimuli for the subjects' perceptual and cognitive abilities. Researchers who work with human infants often encounter similar challenges when studying infant cognition and have developed strategies to overcome these challenges, including using stimuli that capture the infants' attention, determining what tasks are age-appropriate, and using conditioned responses to test discrimination abilities. This paper encourages marine mammal researchers to learn from the research paradigms and techniques used in human infant research and alter them appropriately for the intended study subjects. The conditioned head-turn response, Violation-of-Expectation paradigm, and the help/hinder paradigm have all been used in infant cognition research and show great promise for furthering the current understanding of marine mammal behavior and cognition. In addition, studying a subject's spontaneous behavior can provide valuable insight in areas such as problem solving skills, creativity, and individual differences. Care must be taken to adapt the paradigms and use stimuli to fit each species' perceptual abilities. For example, avoiding a task that requires color discrimination for species that do not possess color vision or using stimuli that fall within a particular species' hearing range are necessary steps in designing an ecologically valid and informative study. Adapting paradigms previously used with human infants can help expand the current understanding of marine mammal communication, cognitive abilities, and social behavior.
\end{abstract}

Keywords - Infant cognition, Marine mammal cognition, Cognition paradigms, Dolphin cognition, Marine mammal behavior

Cognition research with either human infants or non-human animals can be a challenging endeavor. Most of these research subjects have short attention spans, lack verbal abilities, and are simply not interested in some of the research paradigms that are commonly used in adult human cognition research. The goal of this paper is to discuss how the creative solutions (i.e., looking times and behavioral tasks) that are commonly used by human infant cognition researchers to address the challenges of studying infant cognition can be applied to non-human animal communication and cognition research (Winters, Dubuc, \& Higham, 2015). In particular, non-human research may benefit from using conditioned responses to certain stimuli, capitalizing on the subjects' curiosity, choosing the most appropriate task for the subjects, and studying the spontaneous behavior of subjects.

Part of understanding the minds of non-human animals requires learning how animals make sense of their world. There is an abundance of information in any given environment and all individuals have 
limited attentional resources, meaning that they can only focus on and consciously process a finite amount of information at one time. Humans and animals learn to direct their attention to more salient and biologically important stimuli, such as food sources, conspecifics, and potential threats. The "sensitization effect" (Pinske, Hening, Carew, \& Kandel, 1973) is one example that attracts individuals' attention to evolutionarily adaptive stimuli or events. The sight of a predator might be a very salient stimulus for an individual, and thus, attentional resources would be used to process that stimulus over the sight of a food source. Additionally, the individual may become sensitized to a stimulus, such as a predator threat, after having been attacked itself or witnessing a conspecific being attacked. With limited attentional resources, humans have greater neural activation in brain areas associated with emotional processing while viewing fearful faces compared to neutral faces (De Martino, Kalisch, Rees, \& Dolan, 2009). Additionally, neural activation was correlated with an increase in the correct identification of fearful faces, demonstrating how selective attention can benefit an organism.

Certain information is more important for particular species and individuals in specific contexts. For dolphins, sound is a very important sensory modality. Echolocation abilities, for example, are particularly crucial in dark and murky waters where vision is not useful (Au, 1993). Because of this, auditory feedback from echolocation would be very important for detecting predators or prey in conditions of poor visibility; however, in good visibility conditions, a dolphin may devote a greater proportion of attentional resources to visually tracking prey or monitoring a calf's location. Additionally, dolphins may devote attentional resources to conspecifics' whistles to maintain group cohesion in conditions with poor visibility (Janik \& Slater, 1998).

The challenging job of the researcher is to ask and investigate questions that reveal how animals evaluate their changing environment. While the animals may process some information automatically, other information takes effortful processing. The situation is further complicated by the roles that innate abilities versus experience play in developing sensory systems and cognitive skills to make sense of the world. For example, young dolphin calves must learn to effectively produce and use echolocation and whistle vocalizations. Each of these processes may be dependent on different sets of innate abilities, prenatal experience, and early postnatal experience (Kuczaj \& Winship, 2015).

\section{Communication}

Some of the greatest questions facing cetacean researchers today revolve around deciphering the acoustic communication system of whales and dolphins (Janik, 2000; Janik \& Sayigh, 2013; Sayigh, Esch, Wells, \& Janik, 2007). Although this field of study has been persistently increasing over the past few decades and advances in technology are aiding in the effort to decode the acoustic behavior of these animals (Lammers \& Oswald, 2015), there is still a gap in understanding many aspects of cetacean communication. Some questions currently being investigated are: What units comprise cetacean communication systems? (Lammers \& Oswald, 2015); How do young dolphins acquire these units? (Kuczaj \& Winship, 2015); What are the different processes involved in the recognition and production of these units (Cranford, Amundin, \& Krysl, 2015)?

One of the most controversial topics in language research has been the extent to which human language is an innate versus a learned ability (Kuhl, 2000). To answer this question, researchers began studying the development of infants' phoneme recognition abilities (i.e., Altvater-Mackensen \& Grossman, 2014; Werker \& Desjardins, 1995). Phonemes are the smallest unit of human language, and each specific language is composed of a subset of the phonemes from all known languages. Infants are born with the ability to hear and discriminate between phonemes from every language; however, if infants are not exposed to phonemes outside their native language by 10 months of age, they are no longer able to discriminate between similar phonemes to which they have not been exposed (Werker \& Desjardins, 1995). Though adults can learn phoneme contrasts of non-native languages with practice, some phoneme contrasts can be very difficult to learn (Tees \& Werker, 1984; Werker \& Desjardins, 1995; Werker \& Tees, 1984). 
A conditioned head-turn response paradigm was used in a study investigating phoneme discrimination in infants (Werker \& Desjardins, 1995). In this paradigm, children were conditioned to learn that when a repetitive sound they had been hearing changed to a new sound, a stuffed animal would light up and make noise on the side of the room. After infants learned that this event occurred every time the sound changed, researchers presented the infants with sounds that were either two English phonemes or two Hindi phonemes. While 6- to 8-month-old infants whose native language was English were able to discriminate between sets of English phonemes and sets of Hindi phonemes, infants 10 months-of-age and older no longer turned their heads when the Hindi phoneme changed. This study and other studies (e.g., Pons, Lewkowicz, Soto-Faraco, \& Sebastián-Gallés, 2009; Seidl, Cristià, Bernard, \& Onishi, 2009) support the concept of perceptual narrowing, where experience (or lack of experience) with a particular language shapes the perceptual abilities of the developing brain (Maurer \& Werker, 2014). Despite the prevalent use of this paradigm with infants, it should be noted that the stimuli in this paradigm can be interpreted as attention holders and not discriminative stimuli, thus bringing into question the theoretical conclusions that have been drawn from experiments using this paradigm (Weitzman, 2007).

The conditioned head-turn response is a suitable paradigm to use for infants because it does not require a complex understanding of the task and is usually interesting enough to keep the subjects focused on the stimuli and motivated to respond. The same aspects of this paradigm could be applied to research in cetacean communication and the study of communication development in calves. Not only could one use this paradigm to determine the specific units of dolphin acoustic signals, this paradigm also could be used to ascertain how young animals begin to discriminate between meaningful differences in communication sounds. For example, playback recordings of signature whistles from conspecifics have been used to examine long-term memory in dolphins (Bruck, 2013). Playback of whistles could be used in a modified head turn paradigm for dolphins in order to determine what units of sound dolphins discriminate between, similar to phoneme discrimination in infants. Furthermore, longitudinal studies could be conducted to explore how sound discrimination may vary across development in dolphins.

An alternative paradigm to test discrimination is to use paddles and train the subjects to touch a paddle when they perceive a sound as identical to a target (Harley, 2008). Though this can be a very efficient paradigm to use with mature animals that are trained to make this kind of response, young animals may not be trained on many behaviors, thus limiting the subject pool to more mature animals. Additionally, having to learn a multi-step behavior may place more cognitive demands on the subjects, especially if the behavior is non-naturalistic. It is important to consider whether the cognitive demands of a testing paradigm interfere with the results of the study. If the task places too many demands on working memory capacity or requires complex symbolic representation, one could conclude that the subjects do not have a particular capability, when in reality they do.

Engaging the subjects' attention and finding a stimulus or task that motivates them to participate are both important aspects of designing an effective study. Stimuli that dolphins might find engaging are objects with a positive reinforcement history. For example, toys, fish buckets, or familiar trainers are stimuli all likely to capture the attention of the subjects. In studies concerning object permanence, stuffed toys and fish buckets have been used as the target object (Jaakkola, Guarino, Rodriguez, Erb, \& Trone, 2010; Singer \& Henderson, 2014).

\section{Understanding the Subjects' Expectations}

Researchers who study infants are well aware that to engage an infant's attention in a study, the stimuli must be attractive to the infant. The conditioned head-turn paradigm employs a stimulus that has flashing lights, moves, and makes sounds, so that it is a rewarding experience for the infants to turn toward the object at the appropriate time in the experiment. Other studies frequently employ children's toys that are colorful and somewhat familiar to the infant, such as a toy truck or a ball.

While attractive stimuli can incentivize subjects to pay attention to a particular experiment, children's and animals' curiosity can also be used to determine what subjects understand about the world. Looking-time and search paradigms can be designed to take advantage of a subject's tendency to be 
curious about or attentive to a novel or unfamiliar stimulus. If subjects naturally pay attention to unfamiliar or incongruent events, observing which types of stimuli or events capture an individual's attention the most reveals information about how that individual perceives and understands the world.

The violation of expectations (VOE) paradigm (e.g., Baillargeon, Spelke, \& Wasserman, 1985) compares subject responses, as measured by gaze duration, between events that are congruent with past experiences or typical object properties and events that are incongruent with past experiences or object properties. For example, infants gaze longer when viewing a ball that appears to have passed through a solid barrier or a toy truck that appears to float in mid-air after being pushed off a ledge (Stahl \& Feigenson, 2015; see Figures 1 and 2). Both of these scenarios support the argument that infants understand the solidity property of objects and that objects typically fall when not supported. This type of research can reveal at what age different cognitive capacities develop and what cognitive limitations might keep infants from fully understanding a particular scenario by making slight modifications in the parameters of the experiments. The subject's differential attention toward an anomalous scenario indicates what the subject expected to happen and therefore provides insight into the subject's understanding of the world.

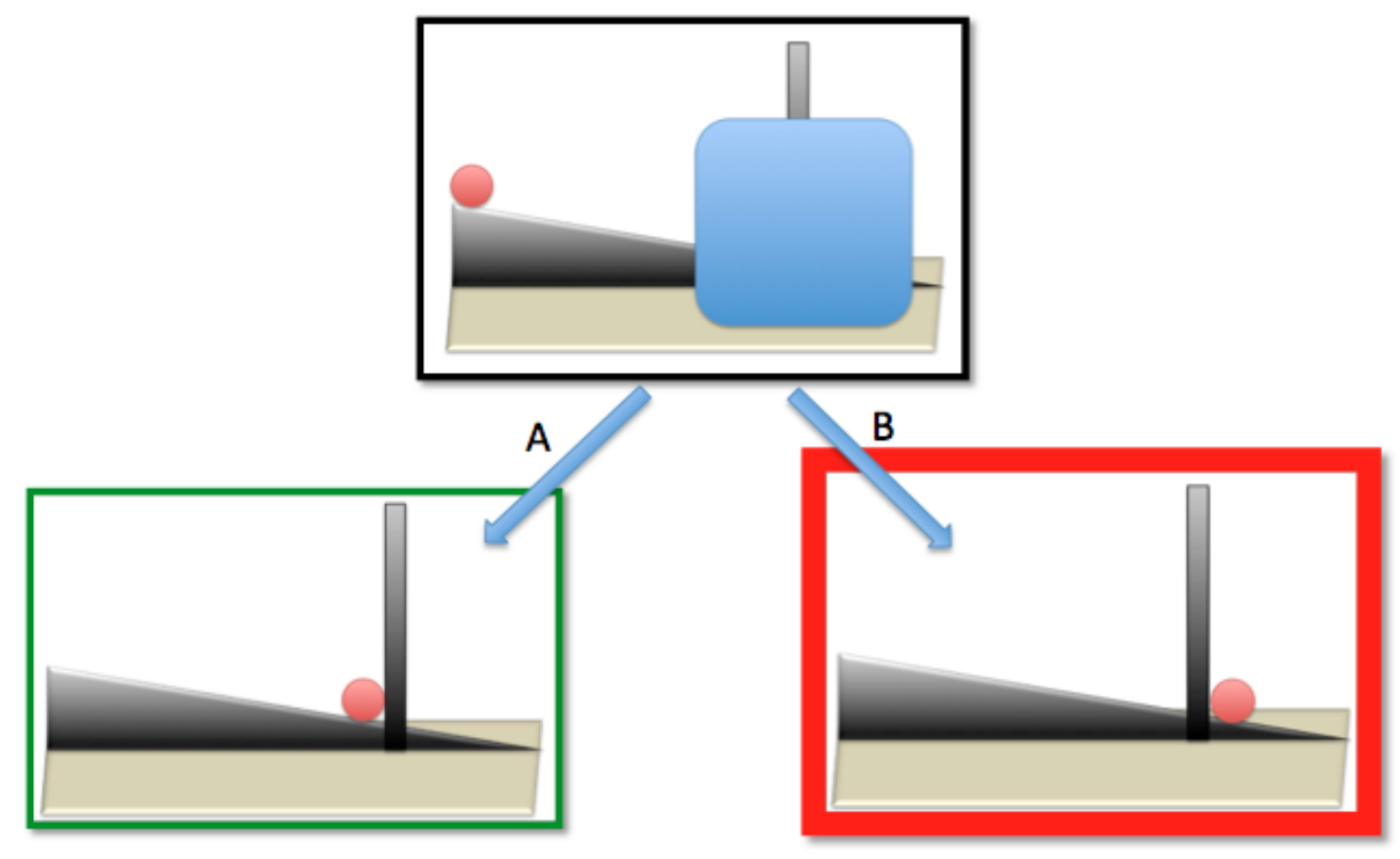

Figure 1. Common paradigm testing infants' understanding of object solidity. After the ball is rolled down the ramp, the screen is moved to reveal either scenario A or B. Scenario A is consistent with the property of object solidity; however, scenario B violates the property of solidity. Infants stare longer at the incongruent event as discussed by Stahl and Feigensen (2015).

Stahl and Feigenson (2015) further expanded on the VOE paradigm by providing the subjects with two objects: one that the infants had just witnessed violates an object property and one that the infants had just witnessed perform the same action without violating an object property. The infants played more with the object that had just behaved incongruently and even performed actions with that object to test the object property that was violated in the incongruent event. The infants were also faster to learn a new property of the object, such as that the object made noise, if they had previously seen the object behave incongruently. This study demonstrates that showing infants an event in which their expectations are violated stimulates curiosity and paves the way for hypothesis testing and learning. This finding matches Piaget's (1952) "moderately discrepant event" in which learning is driven by events that contain both familiar and unfamiliar components. 


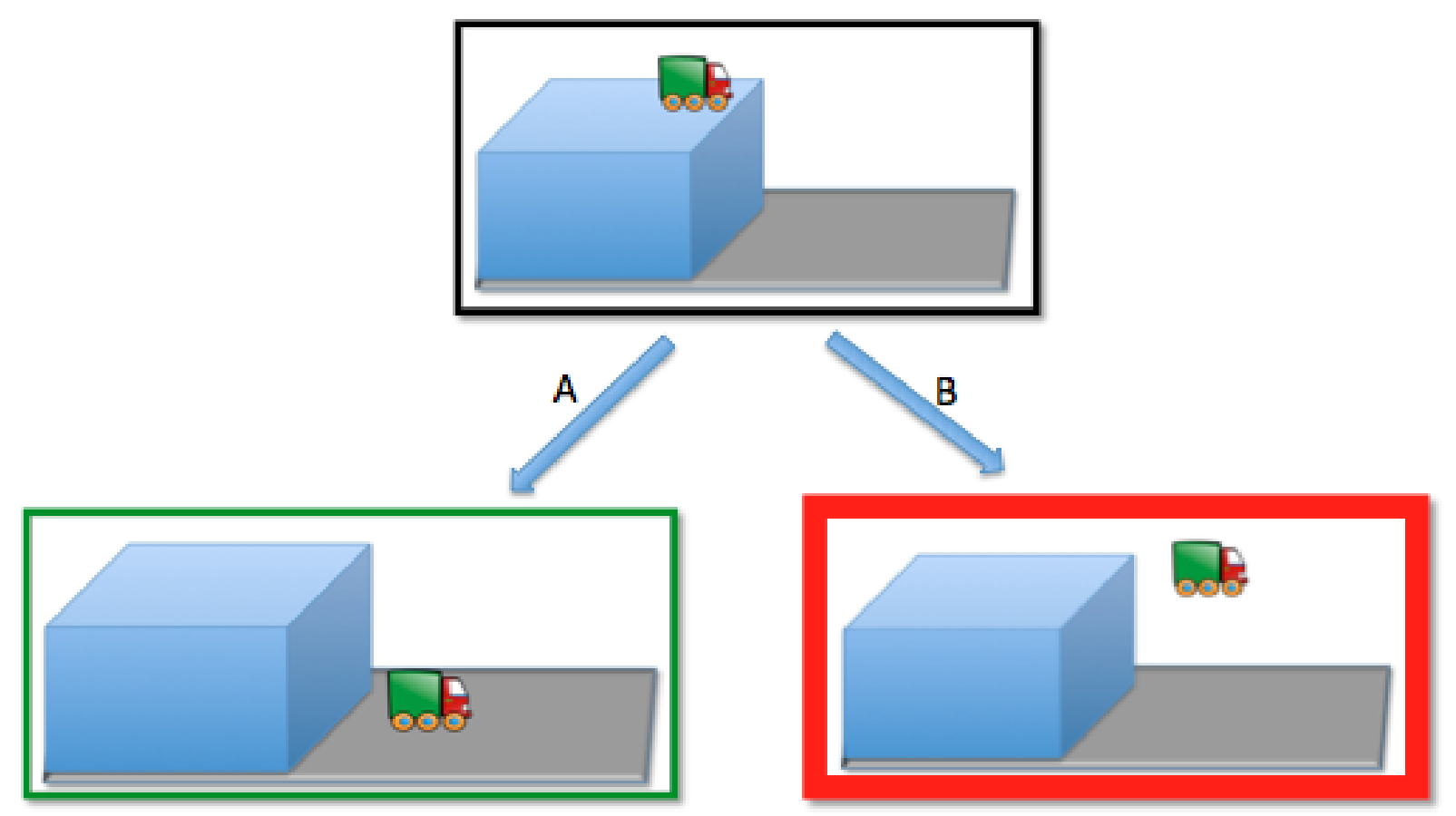

Figure 2. Common paradigm testing infants' understanding of support relations. After the truck is pushed off the ramp, it either falls to the table (Scenario A) or appears to float in mid-air (Scenario B). Infants stare longer at the incongruent event where the truck does not fall when support is removed, as discussed by Stahl and Feigensen (2015).

Potential issues associated with the looking time preference paradigm, of which the VOE is a type, have arisen from studies finding discrepant results with previously published research. For example, the results of Baillargeon et al. (1985) have been attributed to the familiarity or novelty of habituation events as opposed to object permanence (Bogartz, Shinsky, \& Schilling, 2000; Cashon \& Cohen, 2000). Winters et al. (2015) explained that discrepancies between studies are often the product of overinterpretation of results and the lack of proper experimental controls. For example, a longer looking time for a condition in a VOE paradigm may indicate that the subject detects an inconsistency in an objects' behavior based on past events but may not necessarily have a thorough understanding of the concept of object solidity. If these issues are addressed by using proper procedures and not over-interpreting the results, the looking time paradigm is a valid methodology and could be useful for comparisons between species (Winters et al., 2015).

The VOE paradigm has also been used to assess some cognitive abilities in non-human animals, as illustrated by the following examples. Lemurs of four different species looked longer at events in which the number of objects placed behind a screen was not consistent with how many were revealed when the screen was moved (Santos, Barnes, \& Mahajan, 2005). A study investigating rooks found that the subjects looked longer at an event where an object seemed to be floating in mid-air as opposed to when the object was supported by another object (Bird \& Emery, 2010). The concept of gravity and objects appearing to float would be particularly interesting to examine in marine mammals, as the object properties that affect sinking and floating in water are sometimes different than what happens in air. For example, ice cubes fall when dropped in air and an unexpected event would be an ice cube appearing to float in the air after it is released. In contrast, ice cubes float in water, either remaining on the water's surface or floating up to the surface if released below the surface. The unexpected event in this situation would be if the ice were to sink to the bottom of the subjects' habitat instead of floating. Furthermore, dolphins can use echolocation to determine object densities and discriminate between a solid and a hollow object, a property which would affect an object's buoyancy (Au \& Turl, 1991). Given this unique ability, it is likely that some cetaceans could have different expectations regarding a VOE paradigm than, say, terrestrial species would have. 
The VOE paradigm has already been used to test for the capacity of object permanence in dolphins and sea lions (Singer \& Henderson, 2014). Animals were shown a bucket similar to those that hold food during training sessions. This bucket was then placed on a table and an opaque screen was placed in front of it, occluding the object from the subject's view. In the "possible event" condition the screen was rotated $120^{\circ}$ without the bucket becoming visible, and in the "impossible event" condition the screen was rotated $180^{\circ}$, without the bucket becoming visible. Dolphins and sea lions looked longer at events that were incongruent with the concept of object permanence, where the screen would have displaced the object while rotating, indicating that individuals of both species have some conception of object permanence.

A study of this nature with dolphins would first need to assess what events a dolphin finds surprising or what events elicit the subjects' curiosity. Furthermore, behaviors other than gaze duration, such as bubble bursts or vocalizations, may be associated with surprise or curiosity and may be produced more frequently during events that violate the subjects' expectations (Clark, Davies, Madigan, Warner, \& Kuczaj, 2013; Delfour \& Aulagnier, 1997; Hill et al., 2011; Pryor, 1990). A surprising event might also increase the subjects' interest in the object that was involved in that event, which could result in further exploration or manipulation of those objects.

\section{Choosing an Appropriate Task}

Not only is it important to choose stimuli that will help maintain the subjects' attention, but it is also imperative to choose stimuli that do not interfere with the task at hand. For example, infants search more for hidden objects when those objects are familiar than if they are unfamiliar (Shinskey \& Munakata, 2005). Additionally, using a search paradigm results in seemingly conflicting results with a looking paradigm (Keen, 2003). Infants stare longer at events that appear to violate object properties of solidity, such as in Figure 1 when a ball seems to roll past a wooden divide instead of being stopped by the barrier (Spelke, Breinlinger, Macomber, \& Jacobson, 1992). In contrast, if given the task of searching behind a series of doors for a ball that should be resting against a wooden divide (see Figure 3), young children often search at random for the object, not behind the correct door (Berthier, DeBlois, Poirier, Novak, \& Clifton, 2000).

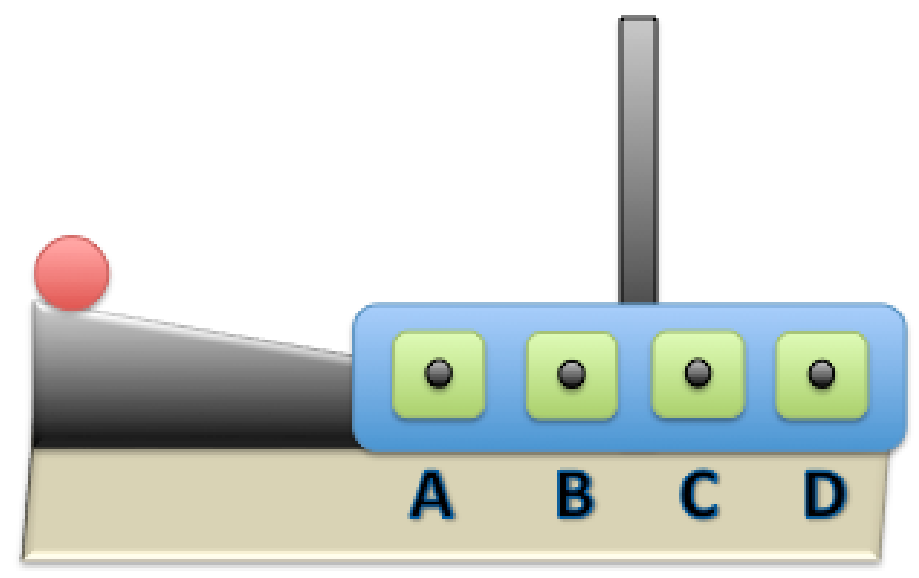

Figure 3. Older infants are commonly tested with this paradigm where a ball is rolled down the ramp and then children must search behind the correct door to find the ball. Though young infants seem to understand object solidity when tested in a looking paradigm, older infants often fail to search behind the correct door in the paradigm shown here. The cue of the barrier between door $\mathrm{B}$ and door $\mathrm{C}$ indicates that the ball should be found behind door B; however, infants may ignore this cue, may not have the ability to focus on the door and the cue simultaneously, may fail to execute the proper motor action, or may not be able to discriminate between the identical doors (Keen, 2003). 
The two paradigms listed above demonstrate that infants' behavior is not always representative of their capabilities, but may be more reflective of the task involved. When infants search for a familiar object, it might be because they have a stronger mental representation of a familiar object, which makes it easier to remember the idea of the object. The second paradigm where toddlers were given the task of searching behind wooden doors for the ball also illustrates a dichotomy between what toddlers seem to understand while looking at a stimulus versus when they are actively searching for the stimulus. As Keen (2003) suggests, this discrepancy may be due to the infants not being able to predict the final location of the object versus just recognizing a discrepancy once it has happened. Other explanations are that the infants are not able to plan their action to open the correct door, or the infants may have difficulty discriminating between the four identical doors. With further study, researchers may find that conflicting results also occur when non-human animals are given different tasks.

Stimuli also should be selected in a way that ensures that the subjects have the sensory abilities to observe the events. For example, some species may not view a video of a person in the same way that they would a live person in front of them due to several factors (D'Eath, 1998). The flicker fusion rate threshold might vary between species, and thus, a video that appears to be continuous to humans may look very different for another species. Dolphins have successfully completed behaviors when presented with a video of a trainer on a television screen. They are also able to complete this behavior provided with only an abstract point-light display (Herman, Morrel-Samuels, \& Pack, 1990). Because the dolphins were able to recognize the stimulus cue from such limited information, it is unclear if the animals understood the representational nature of the human trainers on a screen or were only focused on the hand motions of the trainers in the video. A study on self-recognition abilities in dolphins used a television to display either a live-feed mirror representation of a dolphin or playback of earlier behavior (Marten \& Psarakos, 1995). The dolphins engaged in more open-mouth behaviors in mirror mode and presented marked parts of the body towards the television, indicating that the dolphins were engaging in self-examination rather than social behavior (Marten \& Psarakos, 1995). This study suggests that the dolphins were seeing an accurate representation of themselves on the television screen. Furthermore, a unique touchscreen-like device has been created which allows dolphins to select targets using echolocation beams (Amundin et al., 2008). Although initial testing of the device used basic shapes as targets, this apparatus holds great potential for displaying other visual stimuli for cognitive studies.

Similarly, not all species are able to discriminate between all colors (D’Eath, 1998). For example, dolphins have good vision both above and below water and are sensitive to motion and contrast (Hanke \& Erdsack, 2015); however, dolphins lack short wavelength sensitive cones and thus lack color vision (Fasick, Cronin, Hunt, \& Robinson, 1998). This factor can contribute to a misrepresentation of cognitive abilities if species are tested with colored visual stimuli that must be differentiated for success on a particular task. For example, dolphins are capable of performing matching-to-sample tasks for visual and auditory stimuli (Herman, Hovancik, Gory, \& Bradshaw, 1989); however, if researchers tried to teach subjects a matching-to-sample task with color stimuli, they would fail unless the stimuli differed in some other aspect.

Finally, optimal hearing ranges vary between species which means differences in auditory stimuli may not always be perceived as different or as easily distinguishable (Heffner \& Heffner, 2007). For example, humans have optimal hearing for sounds that range from $250 \mathrm{~Hz}$ to $8.1 \mathrm{kHz}$, while bottlenose dolphins have an optimal range from 20 to $70 \mathrm{kHz}$, illustrating that stimuli of the same frequency might be perceived differently by two different species (Hanke \& Erdsack, 2015; Heffner \& Heffner, 2007).

\section{Social Cognition}

Using a looking or reaching paradigm, researchers who work with human infants also have assessed some aspects of infant social cognition, including examining beliefs about the causes of social events, characteristics of individuals and social groups, and even moral judgments. For example, infants' sense of fairness was assessed via a looking paradigm where infants witnessed a reward being divided 
equally or unequally between two individuals (Sloan, Baillargeon, \& Premack, 2012). Infants at 19- and 21-months-old looked for a longer duration when the reward was divided unequally.

Looking and reaching paradigms also have been used to investigate infants' preferences for others who behave in a positive social manner. For example, social evaluation of others' behavior was demonstrated in 6- and 10-month-old infants in a study where subjects had a looking preference for characters who helped another character compared to a character who hindered another character or a neutral character (Hamlin, Wynn, \& Bloom, 2007). Furthermore, infants as young as 3- and 5-months-old prefer to look at or reach for a puppet that they witnessed behaving prosocially as opposed to one who behaved in an anti-social manner (Hamlin \& Wynn, 2011); however, it should be noted that other studies have resulted in a failure to replicate this original result (Salvadori et al., 2015). A subsequent study found that 16-month-olds tended to match food preferences to others who they have witnessed behave in a prosocial or neutral manner, but did not match food preferences of those who behaved in an anti-social manner (Hamlin \& Wynn, 2012). These studies demonstrate that at a young age, infants are aware of others' social behavior and evaluate others on the basis of social actions.

Other social animals also may evaluate conspecifics or members of other species on the basis of observed behavior. The specific paradigm would need adaptation for each species; however, this basic insight into others' social preferences may possibly inform the current understanding of Theory-of-Mindlike phenomena in non-human animal species. Objects have been used in previous research with nonhuman primates to assess animacy and goal-directedness (Burkart, Kupferberg, Glauser, \& van Schaik, 2011; Kupferberg, Glauser, \& Burkart, 2013; Rochat, Serra, Fadiga, \& Gallese, 2008; Uller, 2004). More recently, the help/hinder and hit/caress paradigm first used with human infants (Premack \& Premack, 1997) was adapted to examine if dolphins attribute dispositional states to moving geometric forms (Johnson et al., in press). The results of Johnson et al. supported that dolphins formed a social interpretation of the objects' actions, due to the dolphins' anticipatory head turns and high arousal behaviors toward dyad-specific displays. Given dolphins' success with this paradigm, future research could adapt other paradigms used with human infants to examine, for example, whether observing prosocial behavior - in objects, puppets, or even conspecifics - would lead dolphins to show food or toy preference-matching.

Dolphins, and other species of cetaceans, live in complex social groups and exhibit cooperation, affiliation, and communication behaviors, which suggest that they may possess advanced social cognition (Johnson, 2015). Males in the wild often form coalitions to increase mating opportunities (Connor, Heithaus, \& Barre, 1999) and dolphins often have complex networks of association with conspecifics (Wells, 2003). Furthermore, dolphins have demonstrated recognition of other individuals (King \& Janik, 2013) and a memory for conspecifics' signature whistles at least 20 years later (Bruck, 2013). Though it is clear dolphins are highly social animals, there is plenty of room to expand research on dolphins' understanding of intentionality, social preferences and conformity, and prosociality, for which the paradigms used in developmental research could be very useful.

\section{Studying Spontaneous Behavior}

Though many of the studies discussed above involve a predetermined experimental procedure with a specific behavior such as gaze or reaching as a dependent measure, research can also capitalize on subjects' spontaneous behavior to study a particular cognitive ability or social behavior. In these cases, the dependent variable can be the spontaneous response of the subjects in a given situation. Studies of infant cognition have also studied spontaneous behavior in situations of free play or where infants are given the option of responding in whatever manner they choose to a set of experimental conditions. For example, studies of attachment style frequently employ the "strange situation" which assesses a child's behavior and interactions with a caregiver in an unfamiliar situation (Ainsworth \& Bell, 1970). Studying the behavior of infants a few days after birth and comparing that to attachment styles at one year of age provides insight into how attachment styles develop (Waters, Vaughn, \& Egeland, 1980). Other studies with infants have focused on child-parent interactions in an effort to understand how children devote their 
attention to people and objects during interactions with others (e.g., Bakeman \& Adamson, 1984). More recently, motion tracking and camera equipment have been used to analyze perceptual and motor patterns of parents and children in a naturalistic setting as a means of understanding how word learning is grounded in the sensorimotor experience (Yu, Smith, \& Pereira, 2008).

Studying the spontaneous behavior of dolphins can yield important information regarding individual development, cognition, and also social behavior. In a study of cross-species social cognition, dolphins' sensitivity to human attention or inattention was assessed by recording their ball tossing behavior to human caretakers while the caretakers were either facing towards or away from the dolphin tossing the ball (Highfill, Yeater, \& Kuczaj, 2016). The results indicated that there are individual differences in dolphins' ball tossing patterns. Depending on whether the human was facing toward or away from the subject, dolphins had consistent individual patterns of how hard they would toss the ball to the human subject and if they tossed the ball at the human's head or the human's body. These results suggest that dolphins may assess the attentional state of humans before deciding when or how to toss a ball to them. Interpreting the results conservatively, the dolphins in this study were attending to the humans' behavior and were using learned situational cues to guide their ball tossing behavior.

Individual differences in dolphins' problem solving abilities also were examined in a study focused on cooperation (Kuczaj, Winship, \& Eskelinen, 2015). Two adult males cooperated in solving the task; however, one adult female was able to open the apparatus by herself in many different ways. This study did not require a trained response and thus allowed the animals to interact with the apparatus and conspecifics without the interference of learned behaviors or the direction of a human caretaker. Though this study did employ a manmade apparatus, its unstructured trials allowed for insight into the subjects' spontaneous social cooperation preferences and individuals' flexibility in using multiple problem solving strategies.

\section{Conclusion}

Previous research in psychology has revealed that infants and non-human animals have more diverse cognitive abilities and a greater understanding of the world than was assumed before such studies were undertaken. Research assessing non-human animals' behavior and cognition has revealed that other species process information through a wide range of sensory modalities, making their knowledge both qualitatively and quantitatively different than humans' knowledge in a variety of different aspects. The challenge of the researcher is to figure out what the subjects know and how this knowledge is acquired. Research techniques used with human infants are not automatically transferable to studies with marine mammals. Care should be taken to examine a particular cognitive ability using more than one paradigm or a paradigm that has been demonstrated to be a valid measure for a particular species or developmental stage. Nevertheless, developmental studies with human infants provide valuable insight on different techniques that may be beneficial to use with non-verbal organisms, such as marine mammals, given that those species also may have limited attention spans and may not fully understand some object properties or perceive stimuli in some modalities. In considering these lessons from the human infant research literature, marine mammal researchers can further the current understanding of marine mammal behavior and cognition.

\section{Acknowledgements}

The authors would like to thank the Society for Marine Mammalogy Conference Methods for Studying Communication and Social Cognition workshop organizers, Denise Herzing and Christine Johnson, for their support of this work. We also would like to thank two anonymous reviewers and Deirdre Yeater for their helpful suggestions and edits to this manuscript. 


\section{References}

Ainsworth, M. D. S., \& Bell, S. M. (1970). Attachment, exploration, and separation: Illustrated by the behavior of one-year-olds in a strange situation. Child Development 71, 49-67.

Altvater-Mackensen, N., \& Grossman, T. (2014) Learning to match auditory and visual speech cues: Social influences on acquisition of phonological categories. Child Development, 86, 1-17.

Amundin, M., Starkhammar, J., Evander, M., Almqvist, M., Lindstrom, K., \& Persson, H. (2008). An echolocation visualization and interface system for dolphin research. Journal of the Acoustical Society of America, 123, 1188-1194.

Au, W. (1993). The sonar of dolphins. New York: Springer-Verlag.

Au, W., \& Turl, C. (1991). Material composition discrimination of cylinders at different aspect angles by an echolocating dolphin. Journal of the Acoustical Society of America, 89, 2448-2451.

Baillargeon, R., Spelke, E., \& Wasserman, S. (1985). Object permanence in five-month-old infants. Cognition, 20, 191-208.

Bakeman, R., \& Adamson, L. (1984). Coordinating attention to people and objects in mother-infant and peer-infant interaction. Child Development, 55, 1278-1289.

Berthier, N. E., DeBlois, S., Poirier, C. R., Novak, J. A., \& Clifton, R. K. (2000). Where's the ball? Two- and threeyear-olds reason about unseen events. Developmental Psychology, 36, 394-401.

Bird, C. D., \& Emery, N. J. (2010). Rooks perceive support relations similar to six-month-old babies. Proceedings of the Royal Society of London B: Biological Sciences, 277, 147-151.

Bogartz, R., Shinsky, J., \& Schilling, T. (2000). Object permanence in five-and-a-half-month-old infants? Infancy, 1, $403-428$.

Bruck, J. (2013). Decades-long social memory in bottlenose dolphins. Proceedings of the Royal Society B, 280 , 20131726. doi:10.1098/rspb.2013.1726.

Burkart, J., Kupferber, A., Glauser, S., \& van Schaik, C. (2011). Even simple forms of social learning rely on intention attribution in marmoset monkeys (Callithris jacchus). Journal of Comparative Psychology, 126, 129-138.

Cashon, C., \& Cohen, L. (2000). Eight-month-old infants' perception of possible and impossible events. Infancy, 1, $429-446$.

Clark, F. E., Davies, S. L., Madigan, A. W., Warner, A. J., \& Kuczaj, S. A. (2013). Cognitive enrichment for bottlenose dolphins (Tursiops truncatus): Evaluation of a novel underwater maze device. Zoo Biology, 32, 608-619.

Cranford, T., Amundin, M., \& Krysl, P. (2015). 2 sound production and sound reception in delphinoids. In D. L. Herzing \& C. M. Johnson, (Eds.) Dolphin communication and cognition: Past, present, and future (pp. 4974). Cambridge, MA: The MIT Press.

Connor, R., Heithaus, M., \& Barre, L. (1999). Superalliance of bottlenose dolphins. Nature, 397, 571-572.

D'Eath, R. (1998). Can video images imitate real stimuli in animal behaviour experiments? Biological Reviews, 73, 267-292.

Delfour, F., \& Aulagnier, S. (1997). Bubbleblow in beluga whales (Delphinapterus leucas): A play activity? Behavioural Processes, 40, 183-186.

De Martino, B., Kalisch, R., Rees, G., \& Dolan, R. (2009). Enhanced processing of threat stimuli under limited attentional resources. Cerebral Cortex, 19, 127-133.

Fasick, J., Cronin, T., Hunt, D., \& Robinson, P. (1998). The visual pigments of the bottlenose dolphin (Tursiops truncatus). Visual Neuroscience, 15, 643-651.

Hamlin, J., \& Wynn, K. (2011). Young infants prefer prosocial to antisocial others. Cognitive Development, 26, 3039.

Hamlin, J., \& Wynn, K. (2012). Who knows what's good to eat? Infants fail to match food preferences of antisocial others. Cognitive Development, 27, 227-239.

Hamlin, J., Wynn, K., \& Bloom, P. (2007). Social evaluation by preverbal infants. Nature, 450, 447-560.

Hanke, W., \& Erdsack, N. (2015). Ecology and evolution of dolphin sensory systems. In D. L. Herzing \& C. M. Johnson (Eds.), Dolphin communication and cognition: Past, present, and future (pp. 49-74). Cambridge, MA: The MIT Press.

Harley, H. (2008). Whistle discrimination and categorization by Atlantic bottlenose dolphin (Tursiops truncatus): A review of the signature whistle framework and perceptual test. Behavioural Processes, 77, 243-268.

Heffner, H., \& Heffner, R. (2007). Hearing ranges of laboratory animals. Journal of the American Association for Laboratory Science, 46, 20-22. 
Herman, L., Hovancik, J., Gory, J., \& Bradshaw, G. (1989). Generalization of visual matching by a bottlenosed dolphin (Tursiops truncatus): Evidence for invariance of cognitive performance with visual and auditory materials. Journal of Experimental Psychology, 15, 124-136.

Herman, L., Morrel-Samuels, P., \& Pack, A. (1990). Bottlenosed dolphin and human recognition of veridical and degraded video displays of an artificial gestural language. Journal of Experimental Psychology, 119, 215230.

Highfill, L. E., Yeater, D. B., \& Kuczaj, S. A. (2016). Catch! Dolphin (Tursiops truncatus) ball tossing to humans is affected by human perspective. Aquatic Mammals, 42, 253-258.

Hill, H., Kahn, M., Brilliott, L., Roberts, B., Gutierrez, C., \& Artz, S. (2011). Beluga (Delphinapterus leucas) bubble bursts: Surprise, protection, or play? International Journal of Comparative Psychology, 24, 235243.

Jaakkola, K., Guarino, E., Rodriguez, M., Erb, L., \& Trone, M. (2010). What do dolphins (Tursiops truncatus) understand about hidden objects? Animal Cognition, 13, 103-120.

Janik, V. (2000). Whistle matching in wild bottlnose dolphins (Tursiops truncatus). Science, 289, $1355-1357$.

Janik, V., \& Sayigh, L. (2013). Communication in bottlenose dolphins: 50 years of signature whistle research. Journal of Comparative Physiology A, 199, 479-489.

Janik, V., \& Slater, P. (1998). Context-specific use suggests that bottlenose dolphin signature whistles are cohesion calls. Animal Behaviour, 56, 829-838.

Johnson, C. (2015). 9 The cognitive ecology of dolphin social engagement. In D. L. Herzing \& C. M. Johnson, (Eds.), Dolphin communication and cognition: Past, present, and future (pp. 230-256). Cambridge, MA: The MIT Press.

Johnson, C., Sullivan, J., Jensen, J., Buck, C., Trexel, J., \& St. Leger, J. (in press). Prosocial predictions by bottlenose dolphins (Tursiops spp) based on motion patterns in visual stimuli. Psychological Review.

Keen, R. (2003). Representation of objects and events: Why do infants look so smart and toddlers look so dumb? Current Directions in Psychological Science, 12, 79-83.

King, S., \& Janik, V. (2013). Bottlenose dolphins can use learned vocal labels to address each other. Proceedings of the National Academy of Science, 110, 13216-13221.

Kuczaj, S. A., \& Winship, K. A. (2015). How do dolphin calves make sense of their world? In D. L. Herzing \& C. M. Johnson (Eds.), Dolphin communication and cognition: Past, present, and future (pp. 49-74). Cambridge, MA: The MIT Press.

Kuczaj, S. A., Winship, K. A., \& Eskelinen, H. C. (2015). Can bottlenose dolphins (Tursiops truncatus) cooperate when solving a novel task? Animal Cognition, 18, 543-550.

Kuhl, P. (2000). A new view of language acquisition. Proceedings of the National Academy of Science, 97, 1185911857.

Kupferberg, A., Glasauer, S., \& Burkart, J. M. (2013). Do robots have goals? How agent cues influence action understanding in non-human primates. Behavioural Brain Research, 246, 47-54.

Lammers, M. O., \& Oswald, J. N. (2015). Analyzing the acoustic communication of dolphins. In D. L. Herzing \& C. M. Johnson (Eds.), Dolphin communication and cognition: Past, present, and future (pp. 107-137). Cambridge, MA: The MIT Press.

Marten, K., \& Psarakos, S. (1995). Using self-view television to distinguish between self-examination and social behavior in the bottlenose dolphin (Tursiops truncatus). Consciousness and Cognition, 4, 205-224.

Maurer, D., \& Werker, J. (2014). Perceptual narrowing during infancy: A comparison of language and faces. Developmental Psychobiology, 56, 154-178.

Piaget, J. (1952). The origins of intelligence in children. New York: Norton \& Company.

Pryor, K. (1990). Non-acoustic communication in small cetaceans: Glance, touch, position, gesture, and bubbles. In J. Thomas \& R. Kastelein (Eds.), Sensory ability of cetaceans (pp. 537-544). New York, NY: Plenum Press.

Pinsker, H. M., Hening, W. A., Carew, T. J., \& Kandel, E. R. (1973). Long-term sensitization of a defensive withdrawal reflex in Aplysia. Science, 182, 1039-1042.

Pons, F., Lewkowicz, D., Soto-Faraco, S., \& Sebastián-Gallés, N. (2009). Narrowing of intersensory speech perception in infancy. Proceedings of the National Academy of Science, 106, 10598-10602.

Premack, D., \& Premack, A. (1997). Infants attribute value to the goal-directed actions of self-propelled objects. Journal of Cognitive Neuroscience, 9, 845-856.

Rochat, M., Serra, E., Fadiga, L., \& Gallese, V. (2008). The evolution of social cognition: Goal familiarity shapes monkeys' action understanding. Current Biology, 18, 1-6. 
Salvadori, E., Blazekova, T., Volein, A., Karap, Z., Tatone, D., Mascaro, O., \& Csibra, G. (2015). Probing the strength of the infants' preference for helpers over hinderers: Two replication attempts of Hamlin and Wynn (2011). PLOS One, 10, 1-10.

Santos, L. R., Barnes, J. L., \& Mahajan, N. (2005). Expectations about numerical events in four lemur species (Eulemur fulvus, Eulemur mongoz, Lemur catta and Varecia rubra). Animal Cognition, 8, 253-262.

Sayigh, L., Esch, C., Wells, R., \& Janik, V. (2007). Facts about signature whistles of bottlenose dolphins, Tursiops truncatus. Animal Behaviour, 74, 1631-1642.

Seidl, A., Cristià, A., Bernard, A., \& Onishi, K.H. (2009). Allophonic and phonemic contrasts in infants' learning of sound patterns. Language Learning and Development, 5, 191-202.

Shinskey, J., \& Munakata, Y. (2005). Familiarity breeds searching: Infants reverse their novelty preferences when reaching for hidden objects. Psychological Science, 16, 596-600.

Singer, R., \& Henderson, E. (2015). Object permanence in marine mammals using the violation of expectation procedure. Behavioural Processes, 112, 108-113.

Sloan, S., Baillargeon, R., \& Premack, D. (2012). Do infants have a sense of fairness? Psychological Science, 23, 196-204.

Spelke, E., Breinlinger, K., Macomber, J. \& Jacobson, K. (1992). Origins of knowledge. Psychological Review, 99, 605-632.

Stahl, A. E., \& Feigenson, L. (2015). Observing the unexpected enhances infants' learning and exploration. Science, 348, 91-94.

Tees, R., \& Werker, J. (1984). Perceptual flexibility: Maintenance or recovery of the ability to discriminate nonnative speech sounds. Canadian Journal of Psychology, 38, 579-590.

Uller, C. (2004). Disposition to recognize goals in infant chimpanzees. Animal Cognition, 7, 154-161.

Waters, E., Vaughn, B., \& Egeland, B. (1980). Antecedents in neonatal behavior in an urban, economically disadvantaged sample. Child Development, 51, 208-216.

Weitzman, R. (2007). Some issues in infant speech perception: Do the means justify the ends? The Analysis of Verbal Behavior, 23, 17-27.

Wells, R. (2003). Dolphin social complexity: Lessons from long-term study and life history. In F. B. M. de Waal \& P. L. Tyack (Eds.), Animal social complexity (pp. 32-56). Cambridge, MA: Harvard University Press.

Werker, J., \& Desjardins, R. (1995). Listening to speech in the first year of life: Experiential influences on phoneme perception. Current Directions in Psychological Science, 4, 76-81.

Werker, J., \& Tees, R. (1984). Phonemic and phonetic factors in adult cross-language speech perception. Journal of the Acoustical Society of American, 75, 1866-1878.

Winters, S., Dubuc, C., \& Higham, J. (2015). Perspectives: The looking time experimental paradigm in studies of animal visual perception and cognition. International Journal of Behavioural Biology, 121, 625-640.

Yu, C., Smith, L. B., \& Pereira, A. F. (2008). Grounding word learning in multimodal sensorimotor interaction. In B. C. Love, K. McRae, \& V. M. Sloutsky (Eds.) Proceedings of the 30th Annual Conference of the Cognitive Science Society (pp. 1017-1022). Austin, TX: Cognitive Science Society. 